\title{
Identifying CNVs in 15q11q13 and 16p11.2 of Patients with Seizures Increases the Rates of Detecting Pathogenic Changes
}

\author{
Gabrielle S. Vianna ${ }^{a}$ Mariana L. Freitas ${ }^{a} \quad$ Valdirene T. de Oliveira ${ }^{a}$ \\ Rafaella X. Pietra ${ }^{a}$ Michele da S. Gonçalves ${ }^{f}$ Patrícia P.O. Rocha ${ }^{a}$ \\ Rejane A.C. Monteiro ${ }^{a}$ Luana C.A. Ferreira ${ }^{a}$ Rosana R. Xaviere \\ Andréia M. Carvalho ${ }^{\text {e }}$ Patrícia R. de M. Lima ${ }^{\text {e }}$ Maria Augusta N.P. Monteiro ${ }^{\mathrm{e}}$ \\ Elvis C. Mateo ${ }^{f}$ Juliana G. Giannettid ${ }^{d}$ Giovana da C. César ${ }^{b}$ Joziele de S. Lima ${ }^{c}$ \\ Paula F.V. Medeiros ${ }^{g}$ Fernanda S. Jehee $^{a}$

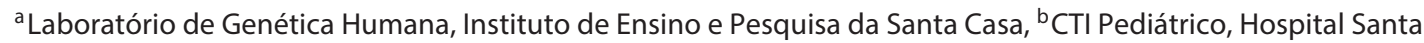 \\ Casa, ${ }^{C}$ Hospital Infantil João Paulo II, and d Faculdade de Medicina da Universidade Federal de Minas Gerais, Belo \\ Horizonte, e Escola Especializada Joana Martins APAE, Santa Luzia, f Setor de Pesquisa \& Desenvolvimento (P\&D) do \\ Instituto Hermes Pardini, Vespasiano, and 9Universidade Federal de Campina Grande, Campina Grande, Brazil
}

\section{Key Words}

Congenital malformations - Developmental delay ·

Intellectual disability · MLPA · Seizures · Submicroscopic

chromosomal changes

\section{Abstract}

Chromosomal changes are frequently observed in patients with syndromic seizures. Understanding the genetic etiology of this pathology is crucial for the guidance and genetic counseling of families as well as for the establishment of appropriate treatment. A combination of MLPA kits was used to identify pathogenic CNVs in a group of 70 syndromic patients with seizures. Initially, a screening was performed for subtelomeric changes (MLPA P036 and P070 kits) and for the regions most frequently related to microdeletion/microduplication syndromes (MLPA P064). Subsequently, the MLPA P343 was used to identify alterations in the 15q11q13, $16 p 11.2$, and $22 q 13$ regions. Screening with MLPA P343 allowed a $10-15.7 \%$ increase in the detection rate of CNVs
\end{abstract}

reinforcing the importance of investigating changes in $15 q 11 q 13$ and 16p11.2 in syndromic patients with seizures. We also demonstrated that the MLPA technique is an alternative with a great diagnostic potential, and we proposed its use as part of the initial assessment of syndromic patients with seizures.

(c) 2016 S. Karger AG, Basel

\section{Introduction}

Chromosomal changes are observed in $\sim 6 \%$ of patients with seizures (SZs), intellectual disability (ID), or developmental delay (DD) and in up to $50 \%$ of patients with epilepsy, congenital malformations (CMs) and dysmorphisms, the so-called syndromic epilepsy [Singh et al., 2002; Bartnik et al., 2012]. Identification of the genetic etiology of these pathologies is essential for an efficient recurrence risk calculation and genetic counseling as well as the determination of possible preventive measures and/or clinical prognosis.

\section{KARGER}

E-Mail karger@karger.com

www.karger.com/msy
(C) 2016 S. Karger AG, Basel

$1661-8769 / 16 / 0076-0329 \$ 39.50 / 0$ 
Some of these changes are observed more often in neuropediatric clinics as they have a stronger association with epilepsy or a greater incidence in the population. They are: monosomy1p36; 4pter (Wolf-Hirschhorn) deletion; ring chromosome 20; 18q deletion, and Miller-Dieker, Angelman, Down, Klinefelter's, and Fragile-X syndromes [Singh et al., 2002; Sorge and Sorge, 2010].

Some other specific changes of the genome also appear to be strongly correlated to SZs, the so-called genomic 'hotspots' that predispose individuals to epilepsy. These include the regions: 1q21, 15q11.2, 15q13.3, 16p11.2, 16p13.11, and 22q11.2 [Chen et al., 2010; de Kovel et al., 2010; Heinzen et al., 2010; Mefford et al., 2010; Mullen et al., 2013].

Thus, the investigation of chromosomal changes in patients with SZs, especially when associated with ID/ $\mathrm{DD} / \mathrm{CMs}$, contributes to the elucidation of many cases.

Despite the established recognition of the role that chromosomal changes play in the genetic etiology of idiopathic epilepsy in Brazil, the genetic component of only a small fraction of patients is determined. This is because the only method offered by the public health system is Gbanding, a technique that is unable to detect the so-called submicroscopic changes (smaller than 5-10 Mb).

Therefore, new methodological approaches which enable the investigation of a greater number of syndromes simultaneously without high costs and with a higher resolution than karyotyping are necessary. The best alternative, undoubtedly, would be microarray analysis as a first line of investigation for patients with ID/DD/CMs/SZs. However, the implementation of this technology is not financially feasible in public health care settings in developing countries.

Therefore, we propose the use of a combination of MLPA kits as an economically feasible diagnostic tool with a great diagnostic potential for the investigation of this group of patients. The MLPA technique is considered a fast, sensitive method capable of detecting chromosomal changes in multiple targets [Schouten et al., 2002], in addition to having lower equipment and reagents costs when compared to techniques to investigate the entire genome.

\section{Patients and Methods}

Patients with ID/DD/CMs/SZs were referred by professionals from various medical specialties, from August 2013 to August 2015.

The criteria for inclusion were: presence of SZs (whenever possible confirmed by additional neurological and laboratory tests), ID or psychomotor DD (associated or not with congenital anoma- lies), and no history of complications during pregnancy and childbirth. All patients were screened by medical specialists, but it was not possible to determine a precise clinical diagnosis. Metabolic disorders and infectious diseases were excluded, to the extent possible.

\section{Sample Collection and DNA Extraction}

Peripheral blood samples were collected from 70 patients. Samples were requested from parents, whenever possible, to establish the mode of inheritance of the detected chromosomal abnormalities.

DNA was extracted with the salting-out method, using the Puregene Blood Core Kit C Qiagen (Qiagen-Sciences, Germantown, Md., USA) according to the manufacturer's protocol.

\section{MLPA}

Initially, the samples were screened using the SALSA ${ }^{\circledR}$ MLPA ${ }^{\circledR}$ P036-E2 and P070-B1/B2 (MRC-Holland, Amsterdam, The Netherlands) for investigation of subtelomeric changes. The SALSA MLPA P064-B2/C1 was used for investigation of the most frequent microdeletions/microduplications. The study was started using the SALSA MLPA P064-B2 to investigate the 1p36 deletion, Williams-Beuren, Smith-Magenis, Miller-Dieker, 22q11.2 deletion, Prader-Willi/Angelman, Alagille, Saethre/Chotzen, and Sotos syndromes. During the study, this kit was updated to the SALSA MLPA P064-C1 which, in addition to the syndromes described above, included the following: Rubinstein-Taybi, WAGR, LangerGiedion syndrome, Wolf-Hirschhorn, Phelan-McDermid, and Cri du Chat syndrome.

For patients without detected changes, the SALSA MLPA P343 was used. This kit contains 52 probes: 9 control probes, three 22q13 probes (chr22: 51,115,059-51,160,754), eleven $16 \mathrm{p} 11.2$ probes (chr16:28,997,152-30,365,260), and twenty-nine 15q11q13 probes (chr15: 25,297,217-32,988,875) with genome coordinates based on hg18/mapview build 36. Although this kit was originally developed for autistic patients, we chose to use it due to its good coverage in the $15 \mathrm{q} 11 \mathrm{q} 13$ and $16 \mathrm{p} 11.2$ regions which, as mentioned above, are significantly altered in patients with SZs.

The reactions were carried out following the manufacturer's protocol using an ABI Veriti Thermal Cycler (Applied Biosystems, Foster City, Calif., USA), and the fragments were separated by capillary electrophoresis in an ABI3730. The display, normalization, and analysis of the results was performed using the GeneMarker ${ }^{\circledR}$ v 2.6.2 software (SoftGenetics LLC, State College, Pa., USA), taking into account the height of the peaks in order to calculate the number of copies of each target sequence. The number of copies considered normal corresponded to a peak ratio value between 0.7 and 1.3 , and for deletions and duplications, a peak ratio $<0.7$ and $>1.3$, respectively. When a change was detected, the reaction was repeated including samples from the parents (when possible) to confirm the result and determine the familial inheritance pattern.

Real Time Quantitive PCR

The real time quantitative PCR (qPCR) technique was used to estimate the breakpoints of the microdeletion, detected using the MLPA P343, observed in a patient. This was performed using the SYBR Green system, with the Fast-Real-Time PCR System 7500 device, as described by Torrezan et al. [2012]. To confirm the extent of the deletion, specific primers were designed for the genes SPNSI, ATP2A1, NPIPB9, EIF3, and NPIPB11 (available upon re- 

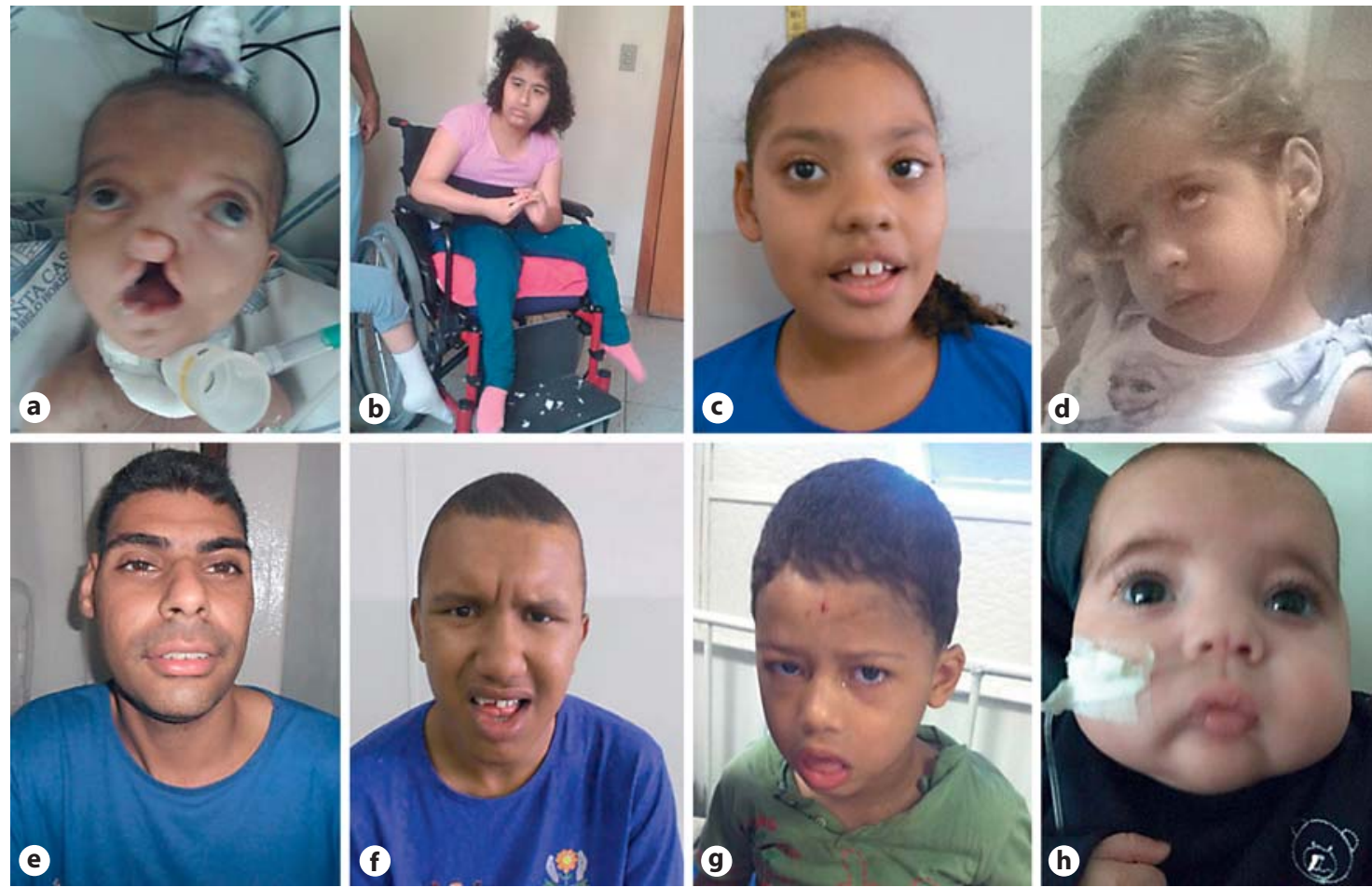

Fig. 1. Photographs of patients with abnormal results detected by MLPA. a Patient 02: del4p16.3. b Patient 04: del15q11q13. c Patient 05: dup15q13 (CHRNA7). d Patient 06: del16p11.2 distal. e Patient 07: dup16p11.2 (CD2BP2). f Patient 08: dup16p11.2 proximal. g Patient 10: del22q11.2. h Patient 11: del22q11.2.

quest), which flanked the deleted probe detected by the P343 kit. To calculate the number of copies of the patient, 10 normal controls (previously tested by SNP array) were used. In addition, all reactions were done in duplicate.

\section{Results}

In total, samples from 70 patients $(60 \%$ men and $40 \%$ women) with ID/DD/CMs/SZs were studied, and 11 changes $(15.7 \%)$ were identified (fig. 1 ; table 1$)$. In the first screening, with the 2 subtelomeric P036 and P070 kits and with the P064 microdeletion and microduplication kit, 7 changes (10\%) were identified. Two unbalanced translocations (del4p16.3/dup8q24.3 and del3p26/ dup20p12.2p13) (2.9\%), 1 deletion in 13q34 (1.4\%), 1 deletion in the Prader/Willi region (15q11.2) (1.4\%), and 3 deletions in 22q11.2 (4.3\%). The remaining 63 patients were screened using the MLPA P343, and 4 (6.3\%) more changes were detected. Three were in the $16 \mathrm{p} 11.2$ (fig. 2) region and 1 in $15 q 13$ (fig. 3 ). Although 3 patients presented CNVs in the $16 \mathrm{p} 11.2$ region, these changes were not identical; therefore, the only CNV that occurred more than once in the sample was del22q11.2, identified in 3 patients and in one of the mothers.

Of the $11(15.7 \%)$ changes found, $9(12.9 \%)$ are definitively pathogenic as they were already well characterized and/or constituted known syndromes. The duplication of the probe located in CHRNA7 was classified as probably pathogenic, and the duplication of the probe located in $C D 2 B P 2$ was considered of uncertain significance, as explained below.

There was no case of disagreement between the results of the 2 subtelomeric kits. They were used together exactly to give greater reliability to the results, avoiding that the diagnostic conclusion be based only on the change in one single probe. Since the reduction in intensity of one probe in the MLPA technique may also be caused by a SNP in the hybridization site, the evidence from 2 distinct probes, changed in the same region, reinforces the deletion hypothesis. In the 2 cases of unbalanced translocations as well as in del15q11.2, the changes were detected by 3 kits, P036, P070, and P064 as these regions were investigated in all of them. 
Table 1. Chromosomal changes detected among the 70 patients analyzed

\begin{tabular}{|c|c|c|c|c|c|c|}
\hline Patient & Phenotypes & Diagnostic kit & CNVs & Abnormal probes & Origin & Pathogenicity \\
\hline 2 & ID/CMs & $\mathrm{P} 036 / \mathrm{P} 070 / \mathrm{P} 064-\mathrm{C} 1$ & del4p16.3/dup8q24.3 & $3(4 p) ; 2(8 q)$ & undetermined $^{\mathrm{a}}$ & pathogenic \\
\hline 4 & ID & P036/P070/P064-B2 & $\begin{array}{l}\text { del15q11q13 (Prader-Willi/ } \\
\text { Angelman syndrome) }\end{array}$ & 6 & de novo & pathogenic \\
\hline 5 & ID/CMs & P343 & dup15q13 & 1 (CHRNA7) & paternal & probably pathogenic \\
\hline 6 & ID & P343 and qPCR & $\begin{array}{l}\text { del16p11.2 distal } \\
\text { (OMIM 613444) }\end{array}$ & $\begin{array}{l}1 \mathrm{MLPA}(L A T) \\
\text { and } 4 \text { qPCR }\end{array}$ & de novo & pathogenic \\
\hline 8 & ID & P343 & $\begin{array}{l}\text { dup16p11.2 proximal } \\
\text { (OMIM 614671) }\end{array}$ & 7 & nonmaternal $^{\mathrm{b}}$ & pathogenic \\
\hline 9 & ID & P064-B2 & $\operatorname{del} 22 \mathrm{q} 11.2(3 \mathrm{Mb})$ & 6 & nonmaternal $^{\mathrm{b}}$ & pathogenic \\
\hline 10 & $\mathrm{DD} / \mathrm{CMs}$ & $\mathrm{P} 064-\mathrm{C} 1$ & $\operatorname{del} 22 \mathrm{q} 11.2(1.5 \mathrm{Mb})$ & 6 & maternal & pathogenic \\
\hline 11 & $\mathrm{ID} / \mathrm{CMs}$ & P064-C1 & del22q11.2 (3 Mb) & 7 & de novo & pathogenic \\
\hline
\end{tabular}

${ }^{a}$ Unable to determine if one parent was a carrier of a balanced rearrangement. ${ }^{b}$ Paternal material not available for analysis.

Based on the analysis from the P343 kit, 3 patients showed abnormal signals of a single probe. In patient 6 , a deletion was identified in the probe located in the LAT gene. When repeating the reaction, the deletion was verified in the patient, and 2 copies of the probe in the parents, again indicating a possible deletion. To confirm the deletion using another technique and to determine its extent, the qPCR technique was used to study the genes flanking the $L A T$ gene. It was observed that the ATPA2 and SPNS1 genes were deleted in the patient, but the NPIPB11, NPIPB9, and EIF3C genes were not deleted (fig. 2). Using the same technique, it again was confirmed that the patient's parents had no deletion in this region. These results allowed the estimation of the deletion size to be between 200 and $400 \mathrm{~kb}$. They further indicate the high probability that the patient has the $16 \mathrm{p} 11.2$ distal microdeletion syndrome (OMIM 613444), mapped in chr16: 28.73-28.95 Mb.

Patients 5 and 7 also had only 1 abnormal probe, located in the CHRNA7 and CD2BP2 genes, respectively. In these cases, however, the changes were duplications, and both were inherited from one of the parents, which rules out the hypothesis of a false-positive result.

\section{Discussion}

SZs are found in many patients with genetic syndromes. However, nongeneticists medical doctors may have difficulty in identifying the possible genetic syn- drome that triggered the phenotypes found. Therefore, the importance of genetic screening in all patients with syndromic SZs is emphasized, mainly looking for CNVs in the regions most commonly altered in this phenotype.

In the present study, chromosomal changes were sought using the MLPA technique with a combination of 4 kits, P064/P036/P070/P343, to determine the genetic etiology of SZs associated with ID/DD/CMs. Chromosomal abnormalities were observed in $15.7 \%$ of the patients (11/70), and only 1 result did not have its meaning clearly identified. Therefore, the MLPA technique proved to be an important tool for screening chromosomal changes in this group of patients. The difference in the present study was including the P343 kit in the screening of patients with SZs. This kit was designed originally to screen autistic patients. However here, P343 increased the rate of $\mathrm{CNV}$ detection in the sample group from 10 to $15.7 \%$.

Rearrangements in $15 q 11.2,15 q 13.3,16 p 11.2$, and 16 p13.11 are more frequently associated with psychiatric diseases such as schizophrenia, autism, and ID [Helbig et al., 2009; Hochstenbach et al., 2009, 2011; D’Angelo et al., 2016]. However, these CNVs also appear to be strongly represented in groups of patients with SZs, indicating a probable common mechanism among these pathologies. Together, these changes are identified in $2.5-3.2 \%$ of individuals with generalized idiopathic epilepsy [Hochstenbach et al., 2011]. Separately, CNVs in 15q11q13 and 16 p 11.2 are present in $1 \%$, and in $1.1 \%$ of patients with epilepsy [Mefford, 2014]. 


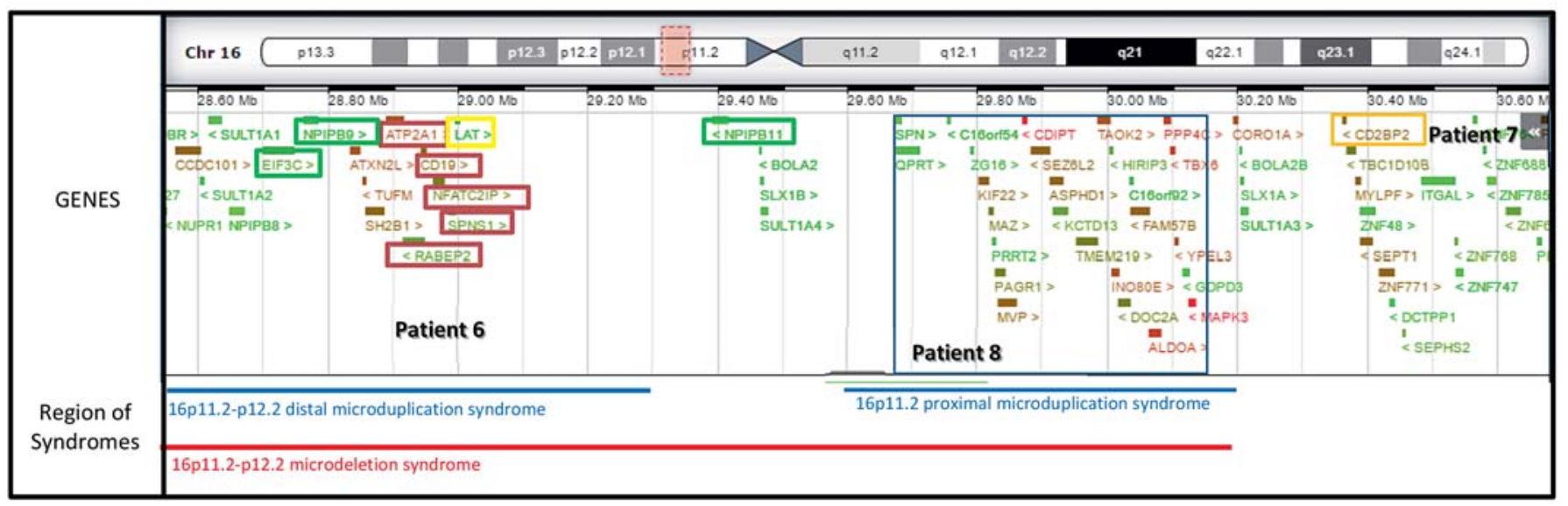

Fig. 2. Genes located in the 16p11.2 region. Patient 6: the yellow box marks the deleted LAT gene detected by MLPA. The red boxes mark the deleted genes detected by qPCR. The green boxes show nondeleted genes. Patient 8: the blue square shows the duplicated genes which correspond to $16 \mathrm{p} 11.2$ microduplication syndrome. Patient 7: the orange box marks the duplicated gene outside the recurring regions of deletion/duplication in 16p11.2. Adapted from DECIPHER (https://decipher.sanger.ac.uk/).

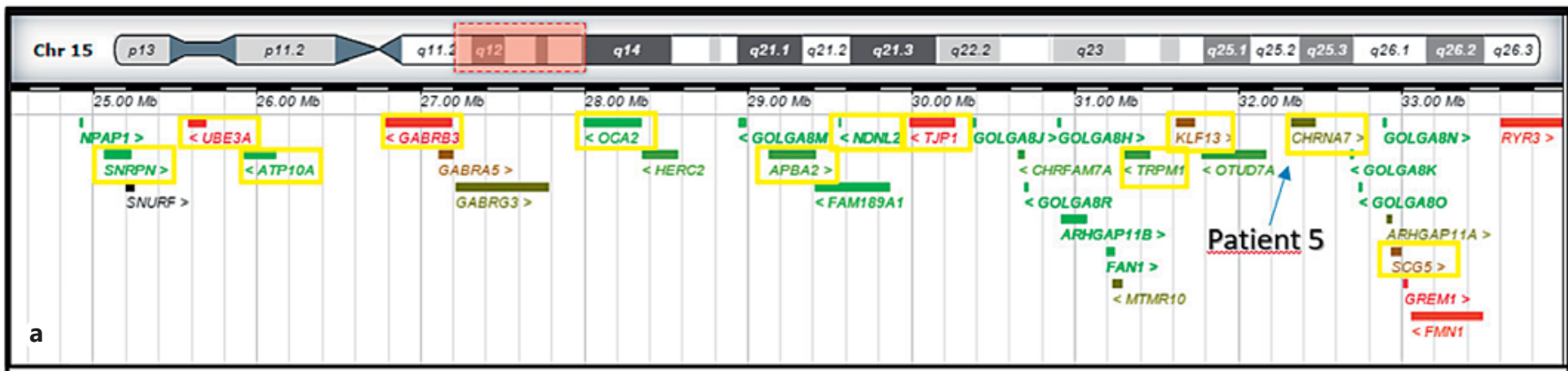

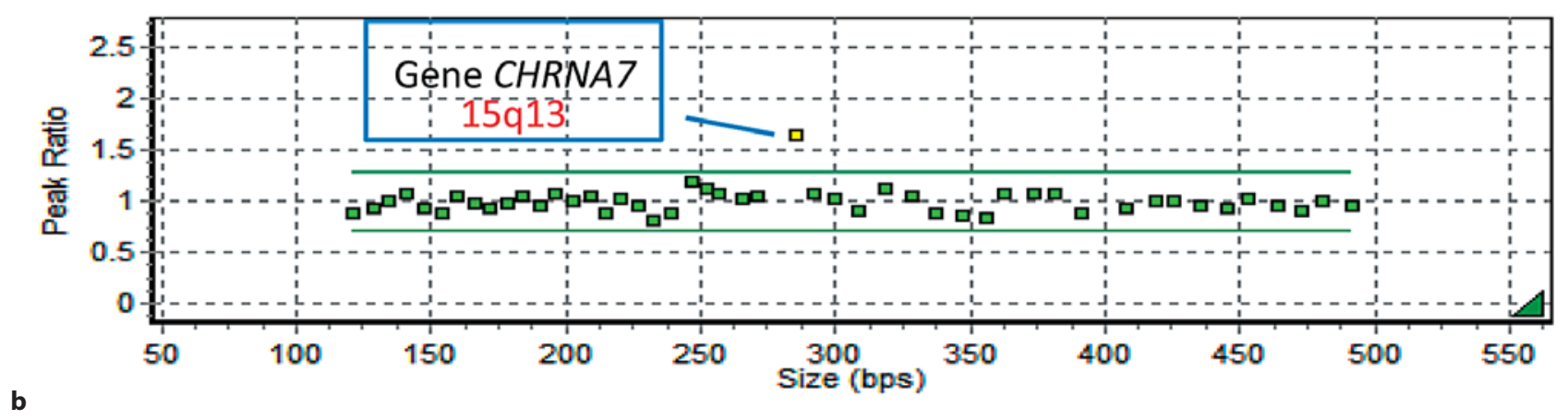

Fig. 3. a Graphical representation of the region $15 \mathrm{q} 11 \mathrm{q} 13$. The yellow boxes highlight the genes that are localized using MLPA P343 kit probes. The arrow indicates the duplicated gene CHRNA7 in patient 5. b MLPA results from kit MLPA P343 in patient 5. Adapted from DECIPHER. 
In the present study, 2 pathogenic or probably pathogenic CNVs were found in $2.9 \%$ of patients in the $16 \mathrm{p} 11.2$ region and in another $2.9 \%$ of patients in the $15 \mathrm{q} 11 \mathrm{q} 13$ region. This reinforces that rearrangements in these 2 regions contribute significantly to the phenotype of patients with syndromic SZs.

Moreira et al. [2014], using the P343 kit, analyzed 531 Brazilian patients with autism. Of these cases, 78 also had SZs. In the group of patients with SZs, 5 (6.4\%) presented CNVs, 3 in 15q13.3 (1 deletion and 2 duplications, including only the CHRNA7 gene) and 2 deletions in 22q13. In the group of patients with SZs, no change was found in $16 \mathrm{p} 11.2$. The authors conclude that changes in $15 \mathrm{q} 13.3$ and $22 \mathrm{q} 13$ are more prevalent among individuals with autism and epilepsy than in individuals with autism alone. The absence of CNVs in 16p11.2 is explained by the phenotypic variability of patients with changes in this region and by the fact that the overlap between autism and epilepsy is not frequently observed [Moreira et al., 2014].

The $16 \mathrm{p} 11.2$ region can be divided into 2 subregions that are frequently altered: a more proximal region of $\sim 520 \mathrm{~kb}$ (chr16: 29,60-30,19 Mb) and the other distal region of $\sim 220 \mathrm{~kb}$ (chr16: 28.73-28.95 Mb). SZs are equally frequent both in patients with deletions and with duplications of the proximal 16p11.2 region [D'Angelo et al., 2016]. The clinical features of patient 8 (fig. 1f), who has the duplication in the above-mentioned proximal region, consist of ID and SZs.

Deletions in the 16p11.2 distal region were initially related to a syndromic form of obesity but have subsequently been described in patients with DD (29.5\%), craniofacial dysmorphisms (22.4\%), behavioral problems (16\%), and epilepsy (9\%). Patient 6 (fig. 1d) has a de novo deletion in the distal $16 \mathrm{p} 11.2$ region. This patient presents some craniofacial dysmorphisms, such as low posterior and frontal hairlines, long eyelashes and synophrys, in addition to scoliosis. The patient developed SZs at 15 months, resulting in neurological regression and then dying at 8 years 5 months, following a long hospitalization to control the SZs.

The third CNV found in the $16 \mathrm{p} 11.2$ region was in patient 7, involving the CD2BP2 (OMIM 604470) gene. Unfortunately, in this case, it was not possible to conduct more experiments to determine the extent of the duplication. Redon et al. [2006] reported 17 duplications in 270 individuals analyzed, suggesting that this region, which includes the CD2BP2 gene, could be considered a polymorphic, nonpathogenic CNV.
The $15 \mathrm{q} 13.1$ microdeletion syndrome was described for the first time by Sharp et al. [2008] and is reported in $1 \%$ of the individuals with generalized idiopathic epilepsy [Helbig et al., 2009]. Duplications in this region are associated with learning difficulties, autism, and SZs [Cook et al., 1997; Thomas et al., 2006]. Although SZs have been proven to be associated with deletions in the CHRNA7 gene, mapped to 15q13.3 [Helbig et al., 2009; Shinawi et al., 2009; Mefford et al., 2010], microduplications involving the CHRNA7 gene, as described in patient 5, were observed in a smaller number of patients with SZs. The duplication detection rate, however, does not seem so low, ranging from 2 to $9 \%$ depending on the study [Szafranski et al., 2010; Moreria et al., 2014; Spreiz et al., 2014]. In addition, many other patients with different variable phenotypes, some including epilepsy, have been described in DECIPHER as having pathogenic or possibly pathogenic duplications, either inherited or de novo, involving the CHRNA7 gene. Thus, although more studies and descriptions of cases are needed to demonstrate the complete pathogenicity of this duplication, it is fairly reasonable to imagine that any dosage variation of the CHRNA7 gene is a predisposing factor for SZs.

In the present study, no CNV was detected in the $22 \mathrm{q} 13.2$ region. The association of changes in this region with SZs is still not very clear, despite some studies [Chen et al., 2010; Striano et al., 2012] suggesting that this is also a candidate region for SZs. The fact that the number of individuals investigated in the present study is not very large may also explain the lack of patients with abnormalities in this region.

Through this study, it was possible to reinforce the importance of investigating microdeletions in $22 \mathrm{q} 11.2$, in patients with syndromic SZs, since this was the most prevalent microdeletion (4.3\%) among the patients involved. The high incidence of SZs among this group of patients has also been discussed in recent studies; e.g., Kao et al. [2004], who investigated a group of 348 patients with $22 \mathrm{q} 11.2$ microdeletion syndrome, found that $7 \%$ presented with SZs. Kao et al. [2004] and Kim et al. [2016] found that among 145 patients studied with the 22q11.2 deletion syndrome, 22 (15.2\%) had SZs.

Understanding of the etiology of ID/DD/CMs/SZs is fundamental for the guidance and genetic counseling of families as well as for the establishment of preventive measures. Another advantage of early identification of the genetic etiology of the patients is the elimination of additional costs arising from the large number of diagnostic procedures used in the attempt to elucidate the cause of the clinical condition of the patients. 
Therefore, considering the diagnostic capacity of the combination of MLPA kits used in the present study, we propose the use of the these kits as part of the initial evaluation of patients with syndromic SZs, along with SALSA MLPA P343 to investigate the 15q11q13 and 16p11.2 regions.

Although the MLPA technique is simpler and less expensive, compared to microarray analysis, the use of 4 diagnostic kits makes the process obviously burdensome. Since the layout of the P064-C1 kit now includes the subtelomeric regions most frequently altered in patients with SZs (del1p36, del4p, and del5p), we suggest using only 1 subtelomeric kit in the initial screening of patients. The follow-up reaction, to confirm and determine the nature of inheritance using parental samples, could be performed with a different subtelomeric kit. This would guarantee a diagnostic result based on 2 distinct probes, which makes the MLPA safer. The use of the P343 kit reinforces both the strong contribution of chromosomal changes in 15q11q13 and 16p11.2 in patients with syndromic SZs as well as the importance of investigating these regions in this group of patients. Therefore, we propose the investigation of microdeletions and microduplications in patients with SZs, including research of these regions using the MLPA technique as an alternative in countries and/or institutions where it is not possible to use microarray analysis as the first line of research to investigate chromosomal abnormalities.

\section{Acknowledgments}

The authors would like to thank the following funding agencies: the Fundação de Amparo a Pesquisa de Minas Gerais/ FAPEMIG, the Coordenação de Aperfeiçoamento de Pessoal de Nível Superior/CAPES, and the Conselho Nacional de Desenvolvimento Científico e Tecnológico/CNPq. We would also like to express our gratitude to João Bosco Pinto Monteiro, president of APAE/SANTA LUZIA-MG; Walter Figueiredo Souza, vice president and superintendent of APAE/SANTA LUZIA-MG, and the patient's families for their participation in the study.

\section{Statement of Ethics}

All patients and their parents or legal guardians have given written consent for the research, and the study has been approved by our ethics committee.

\section{Disclosure Statement}

The authors have no conflicts of interest to declare.

\section{References}

- Bartnik M, Szczepanik E, Derwińska K, Wiśniowiecka-Kowalnik B, Gambin T, et al: Application of array comparative genomic hybridization in 102 patients with epilepsy and additional neurodevelopmental disorders. Am J Med Genet B Neuropsychiatr Genet 159B:760-771 (2012).

Chen CP, Lin SP, Chern SR, Tsai FJ, Wu PC, et al: A de novo $7.9 \mathrm{Mb}$ deletion in 22q13.2 $\rightarrow$ qter in a boy with autistic features, epilepsy, developmental delay, atopic dermatitis and abnormal immunological findings. Eur J Med Genet 53:329-332 (2010).

-Cook EH Jr, Lindgren V, Leventhal BL, Courchesne R, Lincoln A, et al: Autism or atypical autism in maternally but not paternally derived proximal $15 \mathrm{q}$ duplication. Am J Hum Genet 60:928-934 (1997).

D'Angelo D, Lebon S, Chen Q, Martin-Brevet S, Snyder LG, et al: Defining the effect of the 16p11.2 duplication on cognition, behavior, and medical comorbidities. JAMA Psychiatry 73:20-30 (2016).

de Kovel CG, Trucks H, Helbig I, Mefford HC, Baker C, et al: Recurrent microdeletions at $15 \mathrm{q} 11.2$ and 16p13.11 predispose to idiopathic generalized epilepsies. Brain 133:23-32 (2010).
Heinzen EL, Radtke RA, Urban TJ, Cavalleri GL, Depondt C, et al: Rare deletions at 16p13.11 predispose to a diverse spectrum of sporadic epilepsy syndromes. Am J Hum Genet 86: 707-718 (2010).

Helbig I, Mefford HC, Sharp AJ, Guipponi M, Fichera M, et al: 15q13.3 microdeletions increase risk of idiopathic generalized epilepsy. Nat Genet 41:160-162 (2009).

-Hochstenbach R, van Binsbergen E, Engelen J, Nieuwint A, Polstra A, et al: Array analysis and karyotyping: workflow consequences based on a retrospective study of $36,325 \mathrm{pa}$ tients with idiopathic developmental delay in the Netherlands. Eur J Med Genet 52:161-169 (2009).

Hochstenbach R, Buizer-Voskamp JE, Vorstman JA, Ophoff RA: Genome arrays for the detection of copy number variations in idiopathic mental retardation, idiopathic generalized epilepsy and neuropsychiatric disorders: lessons for diagnostic workflow and research. Cytogenet Genome Res 135:174-202 (2011).

Kao A, Mariani J, McDonald-McGinn DM, Maisenbacher MK, Brooks-Kayal AR, et al: Increased prevalence of unprovoked seizures in patients with a 22q11.2 deletion. Am J Med Genet A 129A:29-34 (2004).
Kim EH, Yum MS, Lee BH, Kim HW, Lee HJ, et al: Epilepsy and other neuropsychiatric manifestations in children and adolescents with 22q11.2 deletion syndrome. J Clin Neurol 12: 85-92 (2016).

Mefford HC: CNVs in epilepsy. Curr Genet Med Rep 2:162-167 (2014).

-Mefford HC, Muhle H, Ostertag P, von Spiczak S, Buysse K, et al: Genome-wide copy number variation in epilepsy: novel susceptibility loci in idiopathic generalized and focal epilepsies. PLoS Genet 6:e1000962 (2010).

Moreira DP, Griesi-Oliveira K, Bossolani-Martins AL, Lourenço NC, Takahashi VN, et al: Investigation of 15q11-q13,16p11.2 and $22 \mathrm{q} 13 \mathrm{CNV}$ s in autism spectrum disorder Brazilian individuals with and without epilepsy. PLoS One 9:e107705 (2014).

Mullen SA, Carvill GL, Bellows S, Bayly MA, Trucks H, et al: Copy number variants are frequent in genetic generalized epilepsy with intellectual disability. Neurology 81:1507-1514 (2013).

Redon R, Ishikawa S, Fitch KR, Feuk L, Perry GH, et al: Global variation in copy number in the human genome. Nature 444:444-454 (2006). 
Schouten JP, McElgunn CJ, Waaijer R, Zwijnenburg D, Diepvens F, Pals G: Relative quantification of 40 nucleic acid sequences by multiplex ligation-dependent probe amplification. Nucleic Acids Res 30:e57 (2002).

Sharp AJ, Mefford HC, Li K, Baker C, Skinner C, et al: A recurrent 15q13.3 microdeletion syndrome associated with mental retardation and seizures. Nat Genet 40:322-328 (2008).

-Shinawi M, Schaaf CP, Bhatt SS, Xia Z, Patel A, et al: A small recurrent deletion within $15 \mathrm{q} 13.3$ is associated with a range of neurodevelopmental phenotypes. Nat Genet 41:1269-1271 (2009).

Singh R, Gardner RJ, Crossland KM, Scheffer IE, Berkovic SF: Chromosomal abnormalities and epilepsy: a review for clinicians and gene hunters. Epilepsia 43:127-140 (2002).
Sorge G, Sorge A: Epilepsy and chromosomal abnormalities. Ital J Pediatr 36:36 (2010).

-Spreiz A, Haberlandt E, Baumann M, Baumgartner Sigl S, Fauth C, et al: Chromosomal microaberrations in patients with epilepsy, intellectual disability, and congenital anomalies. Clin Genet 86:361-366 (2014).

Striano P, Coppola A, Paravidino R, Malacarne $\mathrm{M}$, Gimelli S, et al: Clinical significance of rare copy number variations in epilepsy: a casecontrol survey using microarray-based comparative genomic hybridization. Arch Neurol 69:322-330 (2012).

-Szafranski P, Schaaf CP, Person RE, Gibson IB, Xia Z, et al: Structures and molecular mechanisms for common 15q13.3 microduplications involving CHRNA7: benign or pathological? Hum Mutat 31:840-850 (2010).
Thomas NS, Durkie M, Potts G, Sandford R, Van Zyl B, et al: Parental and chromosomal origins of microdeletion and duplication syndromes involving $7 \mathrm{q} 11.23,15 \mathrm{q} 11-\mathrm{q} 13$ and $22 \mathrm{q} 11$. Eur J Hum Genet 14:831-837 (2006).

Torrezan GT, da Silva FC, Krepischi AC, dos Santos EM, Rossi BM, Carraro DM: A novel SYBR-based duplex qPCR for the detection of gene dosage: detection of an APC large deletion in a familial adenomatous polyposis patient with an unusual phenotype. BMC Med Genet 13:55 (2012). 